\title{
Design of an Enhanced TDOA Method for Swept CW Interferences
}

\author{
Hee Won Kang ${ }^{\dagger}$, Deok Won Lim, Moon Beom Heo \\ Satellite Navigation Team, CNS/ATM and Satellite Navigation Research Center, Korea Aerospace Research Institute, \\ Daejeon 605-806, Korea
}

\begin{abstract}
Recently, devices like Personal Privacy Devices (PPD) are being employed to avoid the detection of one's location by GPS, and most PPD transmits swept CW signals. However, signals transmitted from PPD may interfere a precise location system based on GPS. Accordingly, in order to reduce interferences by PPD, a technique to locate an interferer is needed. In order to locate an interferer AOA method and TDOA method are generally used, TDOA method is known to be more accurate than AOA method. Unfortunately, TDOA method has a problem of ambiguity in obtaining measurements of swept CW interference. Thus, this paper design a localization algorithm based on TDOA method that can accurately locate an interferer transmitting swept CW signals by resolving problem of ambiguity. In addition, feasibility of the designed algorithm has been verified by simulation results.
\end{abstract}

Keywords: PPD, swept CW, interference, localization, TDOA

\section{INTRODUCTION}

Developed by the US Department of Defense for military purpose, Global Positioning System (GPS) is a radio navigation system which provides location, speed and time information to users. Recently due to the increasing demand for realtime location information, GPS is utilized in various fields not only in the military but also in private sectors such as the medical, port, and logistics industries. However, devices such as Personal Privacy Devices (PPD) are employed to avoid the detection of one's location by GPS. Available on the internet and much affordable than GPS receiver, PPD can be easily purchased. As the electric power of GPS signals received by a receiver is about - $158 \mathrm{dBW}$, PPD may interfere GPS signals even though it transmits signals with low electric power of about $1 \mathrm{dBW}$. For instance, a truck driver used PPD to avoid detection of his route near Newark International Airport in the US in 2010, and the signals transmitted from the PPD caused a failure in the high-tech instrument landing system of the airport (Pullen et al. 2012).

Received Sep 14, 2012 Revised Oct 20, 2012 Accepted Oct 21, 2012

†Corresponding Author

E-mail: kanghw@kari.re.kr

Tel: +82-42-860-2799 Fax: +82-42-860-2789
Such a failure in a system using GPS may lead to a largescale accident. Thus, recently, some institutions analyzed the type of signals transmitted by PPD and their impact on Global Navigation Satellite System (GNSS) receiver (Kraus, Bauernfeind, \& Eissfeller 2011, Mitch et al. 2011, Grabowski 2012). University of Federal Armed Forces in Germany investigated on interferences such as PPD. According to the study results, it was found that PPD transmitted swept Continuous Wave (CW) signals of the frequency adjacent to GPS L1 signal band, and had a bandwidth smaller than $1 \mathrm{kHz}$. As the signals transmitted from PPD may cause jamming in a precise localization system based on GPS, many institutions are engaged in vigorous research activities to reduce interferences by PPD (Gromov et al. 2000, Balaei, Dempster \& Barnes 2006, Brown \& Reynolds 2010, Cetin, Thompson \& Dempster 2011, Chang \& Huang 2011, Xu \& Trinkle 2011). Most studies, however, were mainly about analyzing signals transmitted by interferers and categorizing them based on their characteristics, so there was no study on localization of interferences. Therefore a study on a technique to locate interference is needed to directly reduce the demage of interferences.

Most typical techniques to locate interference are Array of Arrival (AOA) method which uses the arrival angle of signal and Time Difference of Arrival (TDOA) method 
which uses arrival time differences of signal. AOA method has restrictions that it needs to use array antenna and synchronize phases between RF/IF channels (Niculescu \& Nath 2003), and TDOA method has the restriction of requiring time synchronization between sensors (Smith \& Abel 1987, Chan \& Ho 1994a,b). Moreover, the AOA method has the drawback of causing a huge error if signals are scattered by surrounding environment or sensor is located near a source, this paper using the TDOA method estimates the location of interferences which transmit swept CW signals. However, if the integration time for cross-correlation to obtain a TDOA measurement is longer than sweep time, problem of ambiguity arises as many TDOA measurements are obtained. Because of such ambiguity, general TDOA method cannot be used for localization. Thus, this paper design a localization algorithm based on TDOA method that can accurately locate an interferer transmitting swept CW signals by resolving problem of ambiguity. In addition, feasibility of the designed algorithm has been verified by simulation results.

\section{CHARACTERISTIC OF TDOA MEASUREMENT OF SWEPT CW SIGNALS}

\subsection{Swept CW Signal Model}

Kraus analyzed that PPD transmit swept CW signals, and generally swept CW signals are sinusoidal waves in which frequencies vary by time. Accordingly, the model of swept CW signals is calculated according to Eq. (1) as follows (Kraus, Bauernfeind, \& Eissfeller 2011).

$$
x(t)=a \sin \left[2 \pi\left(f_{0}+k t / 2\right) t\right], \quad\left(0 \leq t<T_{S W}\right)
$$

Here, $a$ is amplitude and has a constant value, $f_{0}$ is initial frequency, $k$ is chirp rate, and $\mathrm{T}_{\mathrm{SW}}$ is sweep time. The Eq. (1) can be changed into Eq. (2) using discrete time.

$$
\begin{aligned}
& x(n)=a \sin \left[2 \pi\left(f_{0}+k n \mathrm{~T}_{\mathrm{SP}} / 2\right) n \mathrm{~T}_{\mathrm{SP}}\right], \\
& \left(\text { when } \mathrm{n} \text { is an integer, } 0 \leq n<\frac{\mathrm{T}_{\mathrm{SW}}}{\mathrm{T}_{\mathrm{SP}}}\right)
\end{aligned}
$$

Here, $\mathrm{T}_{\mathrm{SP}}$ is sampling period, and $B$ stands for bandwidth. The bandwidth can be defined according to Eq. (3).

$$
\mathrm{B} \triangleq \frac{k \mathrm{~T}_{\mathrm{sw}}}{2}
$$

Eq. (2) can be represented as Fig. 1.

\subsection{Characteristics of TDOA Measurement of Swept CW Signals}

In order to locate interferences which transmit swept CW signals by using the TDOA method, TDOA measurement need to be obtained. The TDOA measurement for two arbitrary sensors can be calculated with cross-correlation function, and the function is defined in Eq. (4).

$$
R_{r i}(\tau) \triangleq E\left[s_{r}(t) s_{i}(t-\tau)\right]=\frac{1}{T} \int_{0}^{T} s_{r}(t) s_{i}(t-\tau) d \tau
$$

Here, $s_{r}(t)$ is a signal received at reference sensor, $s_{i}(t)$ is a signal received at $i$ th sensor, which is transmitted signal from an interferer, $T$ is the integration time, and $\tau$ is time delay. As for interference signal, the paper sets to $1 \mathrm{~ms}$ as the period of interference signal cannot be known.

As mentioned in the introduction, when cross-correlating swept CW signals, an issue of ambiguity may arise as the peak of cross-correlation appears repeatedly at every sweep time. Ambiguity arises since sweep time is shorter than integration time. Fig. 2 shows the result of cross-correlating four sensors, while fixing one as a reference sensor with the sweep time of 200 us and the sampling period of $20 \mathrm{~ns}$ in Fig. 1.

It can be seen from Fig. 2 that the peak of cross-correlation

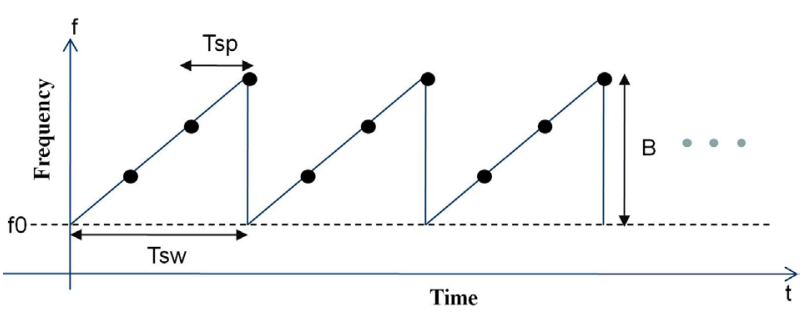

Fig. 1. Swept CW interference signal model.

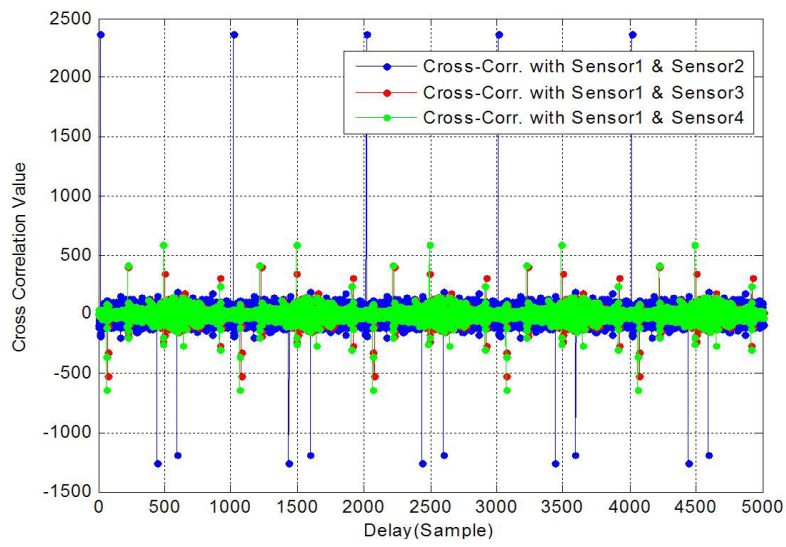

Fig. 2. Cross-correlation value of swept CW signals. 
appears repeatedly at every sweep time. We cannot know true peak of them. Therefore, TDOA measurement cannot be obtained.

\section{DESIGN OF AN ENHANCED TDOA METHOD FOR LOCATION OF SWEPT CW INTERFERENCES}

\subsection{TDOA Measurement Model}

As identified in Section 2, in case of swept CW interference signals, the characteristic of the TDOA measurement has an ambiguity that the cross-correlation value appear repeatedly at every sweep time. Thus, a TDOA measurement model is necessary to track the location of swept CW interferer.

Fig. 3 shows delays of the first peak for samples from 0 to 100. is TDOA measurement obtained by sensor 1 (reference sensor) and sensor 2, and a delay on cross-correlation value which happens first among repeated cross-correlation values. Therefore TDOA measurement of swept CW interference signals can be calculated as Eq. (5).

$$
\tau_{r i}=\Delta \tau_{r i}+N_{r i} T_{S W}
$$

Here, $\tau_{r i}$ is TDOA measurement obtained by reference sensor and $i$ th sensor, $\Delta \tau_{r i}$ is the first TDOA measurement among those repeatedly obtained from reference sensor and $i$ th sensor, and $N_{r i}$ is an integer ambiguity which needs to estimate as ambiguity.

\subsection{Localization Algorithm}

As a localization method using the TDOA method,

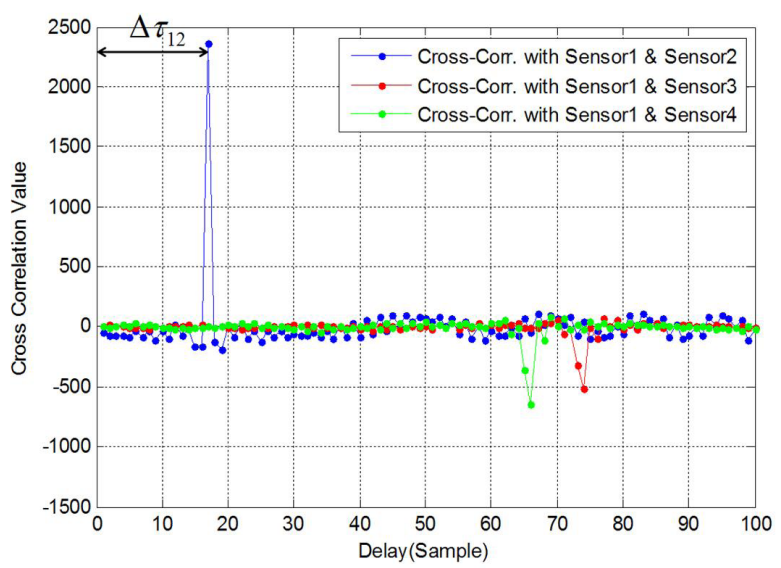

Fig. 3. Delay on initial peak. there are direct solutions and indirect solutions. Spherical Intersection (SX), Spherical Interpolation (SI), Quadratic Correction Least Square (QCLS) are included in direct solutions and Least Squares (LS) is included in indirect solutions. This paper use LS method which is known to find more accurate value compared to direct solutions (Foy 1976, Smith \& Abel 1987, Chan \& Ho 1994a).

Eq. (5) that is equation with TDOA measurement can be expressed as Eq. (6) that is equation with range measurement.

$$
c \tau_{r i}=c \Delta \tau_{r i}+c N_{r i} T_{S W}
$$

Here, $c$ is propagation velocity. A measurement used in the designed algorithm is that is calculated by using the delay obtained from the first peak happened during the integration time. When the number of sensors is $\mathrm{N}, \mathrm{N}-1$ number of equations are gained by substituting $r$ with 1 and $i$ with 2 N in Eq. (6). Thus those equations can be arranged by matrix and can be expressed as Eq. (7).

$$
\begin{aligned}
c\left[\begin{array}{c}
\Delta \tau_{12} \\
\Delta \tau_{13} \\
\vdots \\
\Delta \tau_{1 N}
\end{array}\right] & =\left[\begin{array}{cc}
G_{x 2} & G_{y 2} \\
G_{x 3} & G_{y 3} \\
\vdots & \vdots \\
G_{x N} & G_{y N}
\end{array}\right]\left[\begin{array}{c}
\delta x \\
\delta y
\end{array}\right]-c \mathrm{~T}_{s w} \mathbf{I}_{((N-1) \times(N-1))}\left[\begin{array}{c}
\mathrm{N}_{12} \\
\mathrm{~N}_{13} \\
\vdots \\
\mathrm{N}_{1 N}
\end{array}\right] \\
& =\left[\begin{array}{cccccc}
G_{x 2} & G_{y 2} & -c \mathrm{~T}_{s w} & 0 & \cdots & 0 \\
G_{x 3} & G_{y 3} & 0 & -c \mathrm{~T}_{s w} & \cdots & 0 \\
\vdots & \vdots & 0 & 0 & \ddots & 0 \\
G_{x N} & G_{y N} & 0 & 0 & \cdots & -c \mathrm{~T}_{s w}
\end{array}\right]\left[\begin{array}{c}
\delta x \\
\delta y \\
\mathrm{~N}_{12} \\
\mathrm{~N}_{13} \\
\vdots \\
\mathrm{N}_{1 N}
\end{array}\right] \\
\mathbf{Z}_{S C W} & =\mathbf{G}_{S C W} \boldsymbol{\delta}_{S C W}
\end{aligned}
$$

Here, $G_{x N}$ is $\frac{\hat{x}-x_{N}}{\sqrt{\left(\hat{x}-x_{N}\right)^{2}+\left(\hat{y}-y_{N}\right)^{2}}}-\frac{\hat{x}-x_{1}}{\sqrt{\left(\hat{x}-x_{1}\right)^{2}+\left(\hat{y}-y_{1}\right)^{2}}}, \quad G_{y N}$ is $\frac{\hat{y}-y_{N}}{\sqrt{\left(\hat{x}-x_{N}\right)^{2}+\left(\hat{y}-y_{N}\right)^{2}}}-\frac{\hat{y}-y_{1}}{\sqrt{\left(\hat{x}-x_{1}\right)^{2}+\left(\hat{y}-y_{1}\right)^{2}}},(\delta x, \delta y)$ is delta position, and $\mathbf{I}$ is unit matrix. $\boldsymbol{\delta}_{S C W}$ consisted of delta position and integer ambiguities in Eq. (7) can be calculated by LS method shown in Eq. (8).

$$
\boldsymbol{\delta}_{S C W}=\left(\mathbf{G}_{S C W}^{T} \mathbf{Q}^{-1} \mathbf{G}_{S C W}\right)^{-1} \mathbf{G}_{S C W}^{T} \mathbf{Q}^{-1} \mathbf{Z}_{S C W}
$$

Here, $\mathbf{Q}$ is covariance matrix of measurement error. Thus, to estimate the solution, $\boldsymbol{\delta}_{S C W}$ computed in Eq. (8) add to initial values as Eq. (9). 


$$
\left[\begin{array}{c}
x \\
y \\
N_{12} \\
N_{13} \\
\vdots \\
N_{1 N}
\end{array}\right]=\left[\begin{array}{c}
x_{0} \\
y_{0} \\
N_{(12)_{0}} \\
N_{(13)_{0}} \\
\vdots \\
N_{(1 N)_{0}}
\end{array}\right]+\left[\begin{array}{c}
\delta x \\
\delta y \\
\hat{N}_{12} \\
\hat{N}_{13} \\
\vdots \\
\hat{N}_{1 N}
\end{array}\right]
$$

Finally, the position of an interferer and integer ambiguity can be calculated by repeating from Eq. (3) to Eq. (9) and updating until the magnitude of satisfies specific stop condition.

Fig. 4 shows the flowchart of the designed algorithm in this paper to estimate the position of an interferer which transmits the swept CW signal. This paper presets the stop condition with the magnitude of $\boldsymbol{\delta}_{S C W}$ below 0.001 or repetition over 10 times.

\section{SIMULATION AND RESULTS}

In order to estimate position of interferer by designed algorithm, MATLAB was utilized for computer simulation. Table 1 shows the simulation environment.

Fig. 5 shows cross-correlation value of swept CW signals

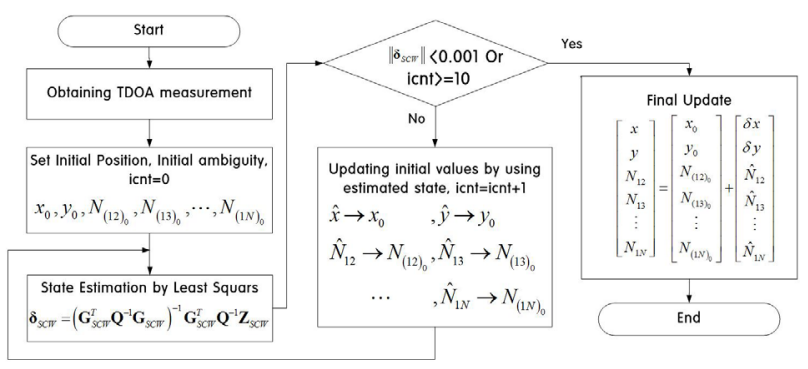

Fig. 4. Flow chart.

Table 1. Simulation environment.

\begin{tabular}{ll}
\hline \multicolumn{1}{c}{ Parameter } & \multicolumn{1}{c}{ Set Value } \\
\hline Sensor arrangement & Arrange four sensors as square form (2000, \\
& 2000), (-2000, 2000), (-2000, -2000), (2000, \\
& $-2000) \mathrm{m}$ \\
Location of interferer & $(868.2,4924) \mathrm{m}$ \\
Type of noise signal & AWGN (Additive White Gaussian Noise) \\
Power of noise signal & $1 \mathrm{~mW}$ \\
Sampling frequency & $5.714 \mathrm{MHz}$ \\
IF frequency & $1.134 \mathrm{MHz}$ \\
Sweep time & $10 \mu \mathrm{s}$ \\
Integration time & $1 \mathrm{~ms}$ \\
Initial position on algorithm & Placeon $(0,0)$ centered on sensor arrangement \\
Number of repeating algorithm & within 10 times \\
\hline
\end{tabular}

created from set sampling frequency and sweep time for the simulation. The TDOA measurement to locate an interferer from several cross-correlation peaks generated at sweep times were found by calculating the integer ambiguities from the designed algorithm, and Table 2 shows actual TDOA, TDOA measurement, and the difference between the two.

It can be seen from Fig. 5 and Table 2 that the designed algorithm can find TDOA measurement by solving the integer ambiguities for happened problem of ambiguity with the cross-correlation peaks repeatedly at every sweep time.

Fig. 6 shows the localization of interferer transmitting swept CW signal by using the designed TDOA method.

As shown in Fig. 6 and Table 3, the designed algorithm can be estimated for location of an interferer transmitting swept CW signal. Thus, It can be confirmed that the designed algorithm can estimate location of an interferer by solving ambiguity of TDOA measurements.

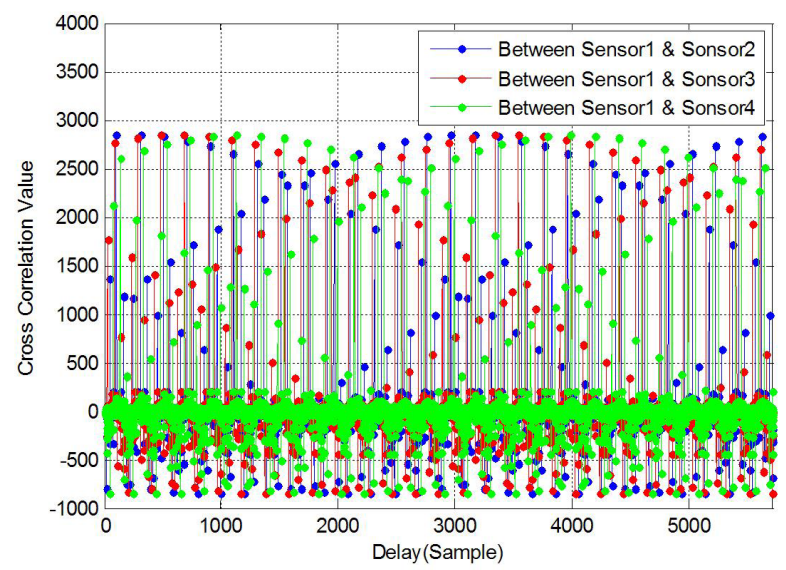

Fig. 5. Cross-correlation value for swept CW signals created for simulation.

Table 2. Actual TDOA, TDOA measurement, error.

\begin{tabular}{lccl}
\hline & $\begin{array}{c}\text { Actual TDOA } \\
\text { (Actual distance/ } \\
\text { minute difference) }\end{array}$ & $\begin{array}{c}\text { TDOA measurement } \\
\text { (Estimated measure of } \\
\text { distance/minute } \\
\text { difference) }\end{array}$ & $\begin{array}{c}\text { TDOA error } \\
\text { (Error of distance/ } \\
\text { minute difference) }\end{array}$ \\
\hline$\tau_{12}$ & $3.202 \mathrm{~ms}(960.5 \mathrm{~m})$ & $3.481 \mathrm{~ms}(1044.2 \mathrm{~m})$ & $0.279 \mathrm{~ms}(83.7 \mathrm{~m})$ \\
$\tau_{13}$ & $14.531 \mathrm{~ms}(4359.2 \mathrm{~m})$ & $14.777 \mathrm{~ms}(4433.1 \mathrm{~m})$ & $0.246 \mathrm{~ms}(73.9 \mathrm{~m})$ \\
$\tau_{14}$ & $12.935 \mathrm{~ms}(3880.5 \mathrm{~m})$ & $13.018 \mathrm{~ms}(3905.4 \mathrm{~m})$ & $0.083 \mathrm{~ms}(24.9 \mathrm{~m})$ \\
\hline
\end{tabular}

Table 3. True position, estimated position, position error.

\begin{tabular}{cccc}
\hline & True position & Estimated position & Position error \\
\hline $\mathrm{x} \mathrm{m}$ & 868.2 & 972.6 & 104.4 \\
$\mathrm{y} \mathrm{m}$ & 4924 & 5034.4 & 110.4 \\
\hline
\end{tabular}




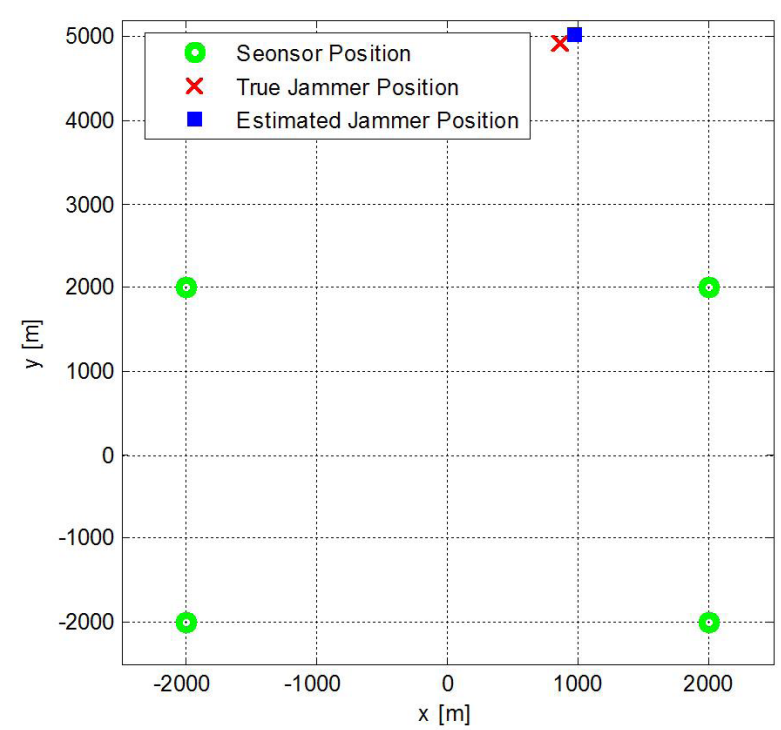

Fig. 6. Results of interference localization using the designed algorithm.

\section{CONCLUSION AND FURTHER STUDY}

This paper modeled the TDOA measurement for swept $\mathrm{CW}$ interference signals, and designed the algorithm to locate position of an interferer transmitting swept CW signal using TDOA method. In addition, we confirmed that the designed algorithm can determine the integer ambiguity to resolve problem of ambiguity and can locate position of an interferer transmitting swept CW interference signal.

A further study will be conducted to analyze changes of performance according to sampling frequency, and design an advanced method with higher accuracy to locate swept CW interferers.

\section{ACKNOWLEDGMENTS}

This work was supported by a grant from Korea Research Council of Fundamental Science \& Technology funded by Ministry of Education, Science and Technology in 2012 [Project: A Study on Satellite based Position Tracking Technology for Calamity Prevention and Public Safety Improvement].

\section{REFERENCES}

Balaei, A. T., Dempster, A. G., \& Barnes, J. 2006, A novel approach in detection and characterization of CW interference of GPS signal using receiver estimation of C/N0, Position, Location, and Navigation symposium,
2006 IEEE/ION, pp.1120-1126.

Brown, A. \& Reynolds, D. 2010, Civil Applications of the GPS Jamming Detection and Location (JLOC) System, Presentations in National Physical Laboratory.

Cetin, E., Thompson, J. R., \& Dempster, G. 2011, Interference Localisation within the GNSS Environmental Monitoring System (GEMS), Proceedings of IGNSS Symposium 2011.

Chan, Y. T. \& Ho, K. C. 1994a, A Simple and Efficient Estimator for Hyperbolic Location, IEEE Transactions On Signal Processing, 42, 1905-1915.

Chan, Y. T. \& Ho, K. C. 1994b, An efficient closed-form Localization solution from time difference of arrival measurements, IEEE Transactions on Acoustics, Speech, and Signal Processing, 2, 393-396.

Chang, C.-L. \& Huang, G.-S. 2011, A Compressive Sampling Approach to Narrowband Interference Elimination for GNSS, Proc. IGNSS Symposium 2011.

Foy, W. H. 1976, Position-location Solution by Taylor-series Estimation, IEEE Transactions on Aerospace and Electronic Systems, AES-12(2), pp. 187-194.

Grabowski, J. 2012, Field Observations of Personal Privacy Devices, Proceedings of ION ITM 2012.

Gromov, K., Akos, D., Pullenm, S., Enge, P., \& Parkinson, B. 2000, GIDL: Generalized Interference Detection and Localization System, Proceedings of ION GPS 2000, pp. 19-22.

Kraus, T., Bauernfeind, R., \& Eissfeller, B. 2011, Survey of InCar Jammers - Analysis and Modeling of the RF signals and IF samples (suitable for active signal cancellation), Proceedings of ION GNSS 2011, pp. 430-435.

Mitch, R. H., Dougherty, R. C., Psiaki, M. L., Powell, S. P., \& O'Hanlon, B. W. 2011, Signal Characteristics of Civil GPS Jammers, Proceedings of ION GNSS 2011, pp. 1907-1919.

Niculescu, D. \& Nath, B. 2003, Ad Hoc Positioning System (APS) Using AOA, INFOCOM 2003, Twenty-Second Annual Joint Conference of the IEEE Computer and Communications, IEEE Societies, 3, 1734-1743.

Pullen, S., Gao, G. Tedeschi, Carmen, T., \& Warburton, J. 2012, The Impact of Uninformed RF Interference on GBAS and Potential Mitigations, Proceedings of ION International Technical Meeting.

Smith, J. O. \& Abel, J. S. 1987, Closed-Form Least-Squares Source Location Estimation from Range-Difference Measurements, IEEE Transactions on Acoustics, Speech and Signal Processing, 35, 1661-1669.

$\mathrm{Xu}, \mathrm{Z}$. \& Trinkle, M. 2011, Interference Angle of Arrival Estimation within the GNSS Environmental Monitoring System (GEMS) using Antenna Arrays, proceedings of IGNSS Symposium 2011. 